\title{
Verzeichnis der Verfasser von Beiträgen
}

Dr. Christoph Averdiek-Bolwin $C A B$

Dr. Thomas Grützemacher $T G$

Dr. Winfried Hierl $W H$

Dr. Elisabeth Kaiser EK

Dr. Sigrid Kletzing SK

Dr. Paul Lerbinger $P L$

Prof. Dr. Matija Denise Mayer-Fiedrich $M D M$

Dipl.-Kfm. Alexandra Freifrau von Ritter zu Groenesteyn $R G$

Prof. Dr. Marcus Riekeberg $M R$

Prof. Dr. Dieter Rohrmeier $D R$

Prof. Dr. Klaus Schredelseker KS

Dr. Georg Thurmayr GT

Prof. Dr. Dieter Witt $D W$ 